\title{
Numerical Simulation of Non-Equilibrium, Ultra-Rapid Heating of Si-thin films by Nanosecond-Pulse Excimer Lasers
}

\author{
A.T. Voutsas \\ Sharp Laboratories of America \\ Camas, Washington, USA \\ avoutsas@sharplabs.com \\ H. Kisdarjono and R. Solanki \\ Oregon Graduate Institute of Science and Technology \\ Beaverton, Oregon, USA \\ hidayat@ece.ogi.edu
}

A. Kumar

Fluent, Inc.

Evanston, Illinois, USA

ak@fluent.com

\begin{abstract}
In this paper we present our work on the numerical simulation of ultrarapid heating (with phase-change) of silicon thin-films, which are irradiated with nanosecond-pulsed excimer laser. Our excimer-laserannealing (ELA) modeling capability is based on a standard finite-element CFD software package, which, however, has been modified to accommodate the specific demands of very rapid heating of thin Si films. In that sense, we've abandoned the traditional equilibrium formulation (i.e. enthalpy method), for phase-change computations, and have adopted a new approach that allows superheated solid and undercooled liquid to exist during the various stages of the heating/cooling cycle. Our model has been successfully applied to predict the shape and temporal evolution of temperature profiles in the case of localized melting of silicon thin-films by excimer laser irradiation. Such scenario corresponds to conditions typically encountered in laser-induced lateral crystallization of a-Si films, a process that has recently attracted attention for the formation of high quality poly-Si films.
\end{abstract}

\section{Introduction}

A number of previous studies and analyses have established that excimer laser annealing (ELA) of thin Si-films is a highly non-equilibrium process in which spatially localized heating with large temperature gradients is produced $[1,2]$. The nature of the physical system, i.e. a very thin Si film typically on top of a thick and insulating $\mathrm{SiO}_{2}$ substrate, further complicates the heat transfer characteristics of the system and introduces two-dimensional thermal and solidification phenomena [2]. For this reason it is improper to simulate excimer laser annealing by applying the same 
type of one-dimensional models developed earlier for laser annealing of bulk $\mathrm{Si}$ wafers [3,4]. The complexity of ELA simulations is further compounded when considering the non-equilibrium nature of rapid heating and cooling, which favors superheating and undercooling phenomena, where solid or liquid exist above or below the bulk melting point, respectively [5]. The idiosyncrasies of excimer laser annealing manifest themselves particularly well in the case of highly localized complete melting of Si thin-films. These conditions favor lateral growth of Si crystals from the edges of the irradiated (molten) domain. During the melting and re-solidification stages a well-defined, sharp interface separates the molten (liquid) silicon from the solidified (solid) silicon. The interface moves laterally (i.e. parallel to the surface of the film) with a velocity that depends upon the superheating of the solid (during melting) and the undercooling of the molten silicon (during solidification).

The crystallization of the spatially localized molten silicon volume is dominated by two competing processes: (i) lateral growth from the edges of the irradiated domain, (ii) homogeneous/heterogeneous nucleation in the bulk. The probability of homogeneous nucleation increases exponentially with the degree of undercooling (i.e. higher undercooling results in higher probability for nucleation). Hence, depending upon the physical characteristics of the domain (i.e. domain width, bias temperature, etc.) and the parameters of the laser irradiation (i.e. energy density, duration of irradiation, etc.) part of the domain may cool sufficiently to trigger homogeneous (copious) nucleation. If this happens before the laterally grown crystal fronts have the chance to intercept each other, the domain will be crystallized partially by lateral growth and partially by copious nucleation. Such a situation is not desirable. Hence, understanding the mode of growth is an important aspect for the successful implementation of the LILC (laser-induced lateral crystallization) process. Furthermore, ability to predict the mode of growth as a function of controllable and/or "noise" parameters can provide a powerful tool to investigate the experimental space in an expeditious and cost-effective manner.

With this impetus we have developed a simulation tool that allows us to properly model LILC process and keep track of critical parameters such as the spatial and temporal variation of the interface, as well as the degree of undercooling in the molten silicon. In this work we report preliminary results obtained from this model showing its utility, strength and application in design of experiments.

\section{Modeling Approach}

In our approach, we have chosen a numerically robust, commercially available CFD code to accomplish this task (FIDAP 8.0 by Fluent, Inc.). This code, however, had to be appropriately modified and further refined to capture the specific features of ultrafast thin-film heating with phase-change. The initial implementation of the code used the "enthalpy method" to determine phase changes and carry the associated computations for the release and absorption of the latent heat over the computational domain. The underlying assumption behind the enthalpy method is thermal 
equilibrium conditions in the modeled system. However, due to the short time scales, the strong localization of the heat source and the large, associated temperature gradients, this assumption is incorrect. In reality, during solidification, the thin film can experience strong undercooling away from the solid/liquid interface. These phenomena imply that the phase of the film is not uniquely determined by the melting point, but it is rather a function of the local degree of undercooling (defined as the difference between the melting point and the prevailing temperature at a given point within the domain). In order to capture these phenomena, we implemented a number of modifications, as suggested by Gupta et al. [6]. This was accomplished via a userdefined subroutine that controls the non-equilibrium absorption or release of latent heat, within the sub-elements of the computational domain. A significant function of this subroutine is the specification of the domains where the extra terms in the heat flow equation [eq. (1)] are valid. Additionally, the subroutine keeps tracks of the location of the solid/liquid interface and its evolution over time.

$$
c_{p} \rho(T) \frac{\partial T}{\partial t}=\frac{\partial}{\partial x}\left(k(T) \frac{\partial T}{\partial x}\right)+\frac{\partial}{\partial y}\left(k(T) \frac{\partial T}{\partial y}\right)+S_{\text {Laser }}(T)+S_{\Delta H_{m}}(T)
$$

where: $c_{p}$ is the heat capacity, $k$ the thermal conductivity, $\rho$ the density. $S_{\text {Laser }}$ is the source term representing the absorbed laser energy and $S_{\Delta H m}$ is the source term that absorbs or releases latent heat. The subroutine computes $S_{\Delta H m}$ by implementing a set of rules to determine exactly the amount of latent heat to be absorbed or released. It also uses a phase function to keep track of the fraction of material melted within a cell of the computational domain. The phase function can have value from 0 to 1 , from complete solid to complete liquid, respectively.

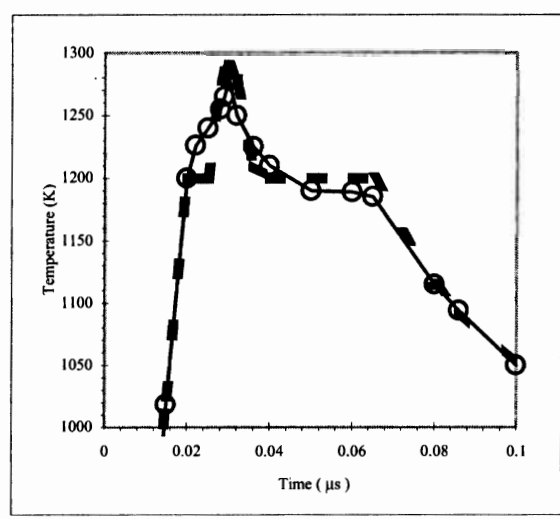

Fig. 1: Temperature history for the domain center from simulations using latent heat function (o) and enthalpy method (- -).

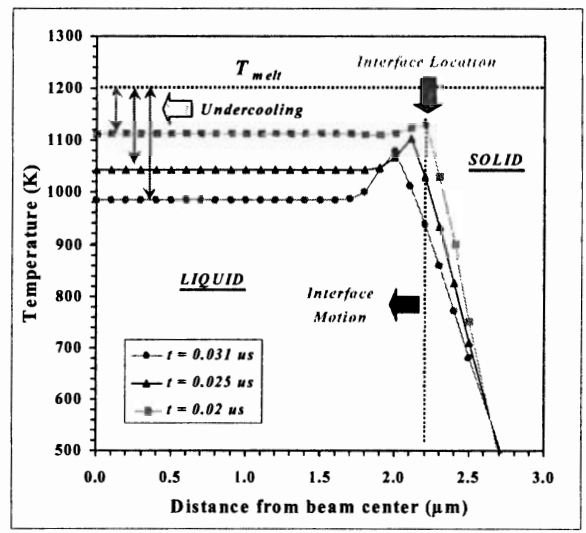

Fig. 2: Spatial temperature profiles across half-beam length at various time intervals during solidification.

As shown in figure 1, when the latent heat function method is used the temperature profiles demonstrate superheating and under-cooling. The enthalpy method does not allow this as is clearly shown by the plateau at the assumed melting point of $1200 \mathrm{~K}$. 


\section{Key Results}

Figure 2 shows an example of the simulated spatial temperature profiles along a $5 \mu \mathrm{m}$ wide region irradiated by a $15 \mathrm{nsec}$ excimer laser $(\mathrm{XeCl}, 308 \mathrm{~nm})$ at $250 \mathrm{~mJ} / \mathrm{cm}^{2}$. Only half-beam profiles are shown due to symmetry (i.e. " 0 " corresponds to the center of the beam). The plot indicates two important features: (1) the precise location and motion of the solid/liquid interface as a function of time (after the laser is switched off). (2) The degree of undercooling and its spatial variation between the edge and center of the liquid region.

Using the degree of undercooling as a measure of the nucleation probability, it is possible to estimate the extent of crystalline lateral growth (LGL) for a given set of irradiation conditions and film parameters. Results of such calculations are shown in figures 3 and 4. Precise modeling of lateral growth is essential for the implementation of LILC process, to obtain very high quality poly-Si microstructures. Our initial predictions agree reasonably well with experimental measurements. More work is currently in progress to improve and refine the predictive ability of our model.

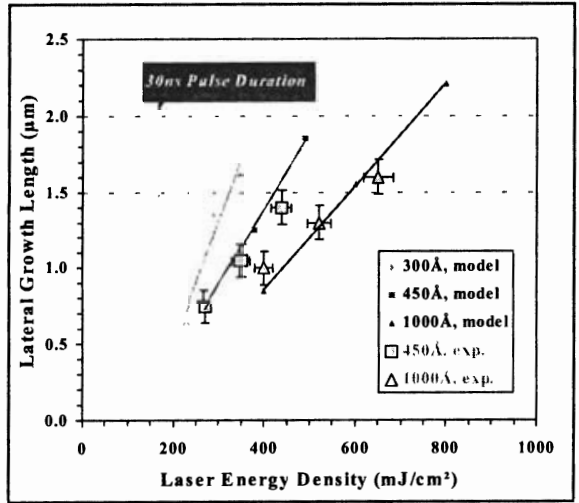

Fig. 3: LGL vs. laser energy density and film thickness.

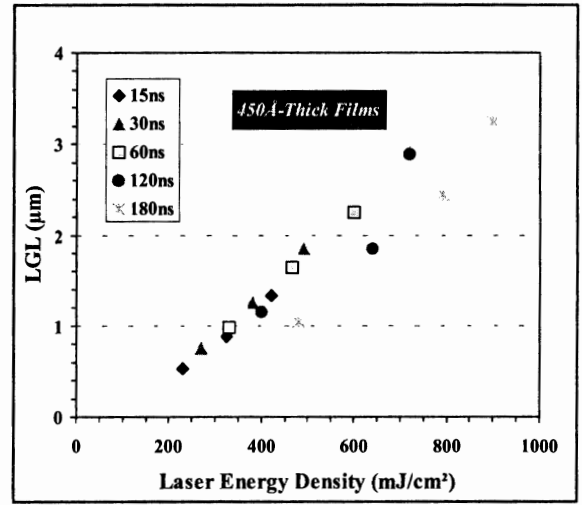

Fig. 4: LGL vs. laser energy density and laser pulse duration.

\section{References}

1. Im, J.S., Kim, H.J. and Thompson, M.O. (1993): Phase transformation mechanisms involved in excimer laser crystallization of a-Si films. Appl. Phys. Lett. 63: 1969-1971

2. Im, J.S. and Kim, H.J. (1994): On the super lateral growth phenomenon observed in excimer laser-induced crystallization of thin Si films: Appl. Phys. Lett., 64: 2303-2305

3. De Unamuno, S. and Fogarassy E. (1989): A thermal description of the melting of c- and a-silicon under pulsed excimer lasers. Appl. Surf. Sci., 36: 1-11

4. Baeri, P., Campisano, S.U., Foti, G. and Rimini, E. (1979): A melting model for pulsinglaser annealing of implanted semiconductors. J. Appl. Phys., 50: 788-797

5. Sposili, R., Im, J.S. (1996): Sequential lateral solidification of thin silicon films on SiO2. Appl. Phys. Lett., 69: 2864-2866

6. Gupta, V.V., Song H.J., Im J.S. (1997): Numerical analysis of excimer-laser-induced melting and solidification of thin Si films. Appl. Phys. Lett. 71: 99-101 